\title{
Se souvenir, réconcilier : les psaumes détournés de Guerre extrême (1999)
}

Ce chapitre parlera des «psaumes détournés » $\left(G E 1_{107}\right)^{1}$ de Guerre extrême, où Tellermann souligne les peines de Paul Celan et leur guerre commune avec la langue, le souvenir et l'Histoire. Si elle met en avant « une langue opaque » (133, $137 ;$ cf. 124), c'est pour mieux faire surgir les sensations et émotions liées au flottement psychique et existentiel que subit tout poète en s'aventurant vers ces zones d'ombre pour accueillir le cri (cf. 25, 50). La guerre intime de Celan avec le passé, son dire façonné par les larmes et le sel des signes à travers lesquels comme en songe il doit nager, seul ou avec autrui, présage ce qui a lieu chez Tellermann : son errance au féminin dans les abîmes de l'inconscient et du langage, sa descente vers la nuit affective peuplée de ténèbres et vers « les phrases affamées de / sens ».2 Souvent, les textes de Celan sont la clé de l'énigme des poèmes de Guerre extrême. Des violences de tout ordre qui hantent la mémoire collective se laissent entrevoir en creux, en tant que traces suggérées par les signes et le phrasé, ainsi que par les rythmes du dire tellermannien axé sur le rituel. Au plan technique, on peut constater d'ailleurs des emprunts aux Psaumes, ces chants écrits par des individus mais dont les reprises et les parallélismes visent le bien collectif, la guérison face aux dangers réels autant que spirituels, la réconciliation avec le mal pour qu'apparaisse de nouveau dans nos bouches un souffle d'au-delà du temps, l'écho du « réconcilié » (128). Au moyen d'allusions et de répétitions, de resserrements et d'épaisseurs, Tellermann parvient à réconcilier la promesse du seuil et la présence du mal, le poème et les cendres, l'ampleur des horizons et la profondeur du souterrain (cf. 9, 89, 128, 131, 145, 147).

1 À une exception près signalée plus loin, toutes les citations entre parenthèses dans ce chapitre viennent de Guerre extrême.

2 Paul Celan, « Hafen » / « Port », [Renverse du souffle], Choix de poèmes réunis par l'auteur, augmenté d'un dossier inédit de traductions revues par Paul Celan, trad. et éd. Jean-Pierre Lefebvre, Paris : Poésie/Gallimard, 2015, p. 248-53 (252 ; nous traduisons). Cf. Paul Celan, Enclos du temps / Zeitgehöft, trad. Martine Broda, Paris : Clivages, 1985, p. 39-45 (recueil non paginé auquel nous attribuerons des numéros de page).

(C) AARON PREVOTS, 2022 | DOI:10.1163/9789004499683_003 
Comme le signale Gilles Jallet, ce recueil est caractéristique de la poésie d'Esther Tellermann parce qu'il s'ouvre d'emblée aux lieux et au temps. ${ }^{3}$ En effet, Tellermann aime à observer des lieux concrets, à découvrir au gré de ses pérégrinations « des continents, des peuples ». En tant que poète, elle trouve au fur et à mesure les moyens de communiquer par le biais de la forme ce qui relie les lieux, la dimension collective et historique du vécu et l'atemporel mythique que communiquent les signes. ${ }^{4}$ L'une des hypothèses principales de la présente étude est que Paul Celan deviendra dans son œuvre celui qui l'accompagne le plus fidèlement aux niveaux affectif et linguistique. L'Autre qui infléchira le poème vers l'Histoire et le dialogue, c'est lui. Or, Tellermann ne le copie pas, loin s'en faut. Il s'agit plutôt de le suivre dans la brèche qu'il ouvre dans la langue, dans le passage étroit qui mène à la communion avec soi et l'Autre, la langue et les origines. De même, Celan en tant que lecteur des Psaumes nous apprend à confronter l'espoir et le désespoir, à reconnaître nos peines et le sacré qui nous réconforte. ${ }^{5} \mathrm{Il}$ met en lumière la manière dont ses mots en tant que pierres fragiles pourront nous aider à bâtir un avenir partagé. En lisant Guerre extrême, gardons en esprit ces liens, ces problématiques, ces profondeurs. Remarquons en particulier des moments où ce recueil fait écho à Enclos du temps et à Renverse du souffle, écrits tous les deux vers la fin des années 1960 et publiés respectivement en traduction française en 1985 et 2003. Le but de notre lecture attentive sera triple : comprendre l'énigme, faire retentir des prières et suivre un mouvement vers autrui.

Considérons tout d'abord le fait qu'il n'y a pas de guerre précise à chercher. Tellermann s'attaque aux mots, aux silences qu'ils recouvrent, aux détresses profondes de lâme qu'incarne l'étroitesse du dire. ${ }^{6}$ Certes elle a composé Guerre extrême au Vietnam, ${ }^{7}$ mais c'est un livre à multiples facettes, dont l'ambiguïté implique le lecteur averti. Nous avons affaire à une locutrice dont on

3 Gilles Jallet, « Esther Tellermann : Guerre extrême », Le Nouveau Recueil 54 (mars-mai 200o), p. $168-70$.

4 Aaron Prevots, «Entretien avec Esther Tellermann », op. cit., p. 102 : « Lorsque je suis revenue avec le manuscrit Guerre extrême, composé au Vietnam, j'ai compris que je pouvais aussi tendre dans la forme poétique à une réflexion sur le mythe et l'Histoire, mais une histoire fantasmée bien sûr, passée au prisme de l'inconscient et de l'oubli. [...] [J]e pouvais narrer une histoire collective appréhendée dans les ruines, les restes des civilisations, m’approprier imaginairement une mémoire collective. Je cherchais une alliance avec la langue, un pardon possible à la violence que furent pour moi les déportations et les persécutions des juifs pendant le nazisme en Europe. »

5 Cf. Robert Alter, The Book of Psalms. A Translation with Commentary, New York: W. W. Norton \& Co., 2007, p. xiii et p. $120 n 19$.

6 Ibid., p. $491 \mathrm{in} 8$.

7 Aaron Prevots, «Entretien avec Esther Tellermann », op. cit., p. 102. 
peut dire que l'âme est en train d'« orager », qui nous apprend « comment on sombre / et devient vrai » (cf. GE 83, 129). ${ }^{8}$ À l'instar de Celan, nous nous mettons à l'écoute d'un bruit des tempes « où bat le cœur », ${ }^{9}$ ainsi que du bruit sec de doigts qui traversent « l'abîme » et de celui, insaisissable, de tout ce qui erre dans notre souvenir, « lécho irréfutable / de toute ombre gagnant $» .{ }^{10}$ Notre attention s'accorde à de telles ténèbres, réclame de telles vérités, tellement les textes liminaires par lesquels commence Guerre extrême - gnomiques, oraculaires, dépouillés, quelquefois monosyllabiques - nous contraignent au silence méditatif. S'y annonce la prise de conscience d'« écueils », de limites humaines, d'une dénudation «jusqu'au temps», mais aussi d'un Nous collectif qui se servira rituellement des gestes de la parole et regardera ce que le poète lui montre, afin d'accepter ces limites, de renouer avec autrui et les lieux « d'où nous fûmes issus » et de résister à la « [f]atigue » et à l'usure du regard que représentent les «gazes trouées » $(9-12,17)$. Les découvertes seront donc partagées. Le Je lyrique nous plongera dans des « [s]ommeils parallèles » (125) : ceux des poètes-témoins ainsi que des êtres qui nous furent chers et qui, comme en rêve, se rangent à nos côtés. ${ }^{11}$ Creuser, ce sera devenir (cf. 103, 117). Songer, ce sera se heurter à ce qu'il reste de «lourd » (125) dans la parole, l'inconscient, le dire, puis brûler les « encens » (124) pour exprimer sa désorientation, souffler, puis en plein abîme repartir (cf. 129, 133, 141-47).

Regardons aussi comment un modus operandi se met en place. Premièrement, Tellermann tend vers le récit en mettant les deux vers manuscrits à la page 7 : « Ils finissaient sous des bâches / ou dans les cartons des villes souterraines. » Or, cela sert plutôt d'épigraphe, s'ouvrant comme un travelling sur les fragments de vers qui suivent et sur le cadre aux contours spatiotemporels imprécis et pleins d'ombre. On abordera la mort, mais de biais. Il faudra patienter avant que de tels fils narratifs ne resurgissent. Deuxièmement, on frôle l'énigme mais aussi l'« urgence » (11). Les instantanés de ce qui traverse

8 Paul Celan, « Mon / âme inclinée vers toi », Enclos du temps / Zeitgehöft, op. cit., p. 51.

9 Paul Celan, « Dans la plus lointaine », Enclos du temps / Zeitgehöft, op. cit., p. 25. La comparaison de notre part relie deux références celaniennes. Dans Enclos du temps, il s'agit d'un « profil » qui est « renforcé de fibres du rêve », une « sécrétion du sommeil » qui prend des allures de revenant quelque peu mystique et qui n'a qu'une seule tempe. Dans « Attaque de violoncelle », [Renverse du souffle], Choix de poèmes, op. cit., p. 267-69 (267), il est question du souffle creusant dans un livre ouvert par le bruit de la pensée. Chez Tellermann comme chez Celan, la versification et les sonorités évoquent par moments un tel acheminement du souffle et le pouvoir affectif de la pensée. op. cit., p. 43 et p. 45 .

Paul Celan, « Dans la plus lointaine », Enclos du temps / Zeitgehöft, op. cit., p. 25. 
la langue, les lieux et la pensée feront une place pour l'indicible. Un certain aveuglement sera de mise, pour mieux faire valoir le réveil et la réflexion : « vers bride le simulacre » (85). Lutter avec le sens qui «nous contraint » (12) permettra à Tellermann d'explorer l'humain au sein de l'Histoire et du deuil « corps », « Palais », « Peaux », « L'odeur » (9) - sans tomber dans le banal et les redites de la simple représentation. Chaque trou dans la représentation facilitera l'accès à la durée, à ce qui semble s'éteindre mais peut donner sur du « lumineux » (140; cf. 135). Troisièmement, toute une gamme d'énoncés baliseront le terrain à parcourir. Comme le suggèrent les textes liminaires, il $\mathrm{y}$ aura un équilibre entre le fragment de thème et le continuo, le mystère et le moiré illuminant de l'étoffe. Parsemé d'allusions mais aussi de vers et de mots plus transparents, le texte sera comme les « sureaux » qu'il s'agit de «sav[oir] » et d'« attend[re] » $(22,100)$ : tantôt remarquable comme une fleur en train de s'épanouir, tantôt surprenant à cause des sens cachés sous la peau de fruits acides noirs. Il y aura des « symboles / clos », mais qui nous berceront (94; cf. $71,114)$ pour nous mettre à la recherche de « définitions » (93). Aussi saccadé que soit le début de Guerre extrême, rien de plus universel, par exemple, que le fait d'être « 2 » au « centre » de ces découvertes (11).

Comprendre les allusions dans la première séquence, « Terre mentale », nous aidera à participer à la prière dont il est question. Ce titre nous situe non pas dans un jeu abstrait de signes sans fond, mais dans l'envie d'accompagner autrui dans une lecture passionnée « des formules » (17), de veiller ensemble sur le langage et l'Autre, de glaner des restes et de les examiner de près. Nous devons noter l'incommensurable et le très concret, ${ }^{12}$ nous rendre compte d'un réel qui nous résiste à cause de l'événement qui le mine. La terre parfois sans poids de l'œuvre de Celan dont il est souvent question, son « Ungrund $»^{13}$ ou « abysse », correspond au chagrin sans fond qu'a pu causer la Shoah. Celan est en quelque sorte le géographe qui a mesuré cet impossible pour en établir la

Jean-Marie Gleize, «Esther Tellermann », Dictionnaire de poésie de Baudelaire à nos jours, éd. Michel Jarrety, Paris : PUF, 2001, p. 816-17 (817) : « [I]l s'agit d'une guerre sans fin. La difficulté de la poésie d'Esther Tellermann provient sans doute de notre capacité à mesurer, c'est-à-dire à comprendre le poids de chacun de ces deux mots "guerre extrême". Il s'agit très exactement d'une poésie d'expérience. »

Paul Celan, « Eingeschossen » / «Projeté », Enclos du temps / Zeitgehöft, op. cit., p. 26-27. Le mot « Ungrund », qui signife dans Enclos du temps à la fois le sans-terre, le sans-raison, le sans-fondement, la matière trouée et l'errance qui y a lieu, vient de la littérature et la philosophie mystique allemande à partir du XII e siècle. Il se réfère chez Jacob Böhme (1575-1624) à l'origine du monde en tant qu'abîme, néant, indétermination, absence incompréhensible. Cf. David König, Le Fini et l'Infini: l'odyssée de l'absolu chez Jacob Böhme, Paris : Cerf, 2006. 
mappemonde. Tellermann, à sa suite, vogue dans le «fleuve» du souvenir, se rappelle les « guerres extrêmes » au pluriel, vise comme lui et Rilke avant lui ce qui « fleurit en pauvre lieu » $\left(19,48,122 ;\right.$ cf. 64). ${ }^{14}$ Comme chez Celan, les « formules » de Tellermann explorent une tension entre le songe qui embrasse l'éternité et la nuit inénarrable de l'Histoire qui affecte toujours le présent (cf. 64, 103, 139). Elles parlent de la « prière » qui semble impossible mais qui se poursuit tout de même, voire du « Dieu » qui indique la présence de nos semblables et des « baumes et racines » que peut planter, tels ce Dieu et sa parole, la parole poétique $(41,62,90)$. L'impossible n'est pas seulement une tristesse qui crève le cour, mais aussi la montée d'une certaine folie lorsqu'on y est confronté. Il convient de remarquer les failles dans la langue, puis de respecter le silence pour capter les chants brisés d'en bas, les « respir[er] », les «tiss[er] [...] en lieux / neufs », les replanter « jusqu'au dernier nœud / du souffle » $(29,80,132 ; \mathrm{cf} .24,29,50,64)$. Il y a un va-et-vient entre les choses observées, la « scène mentale » (64) de l'imaginaire et sa mise en mots, et le dialogue avec Celan, sans que ces niveaux sémantiques ne soient des cases étanches. Penser à Celan, en somme, nous rappelle le rôle que joueront dans Guerre extrême l'affect, l'inquiétude, l'intersubjectivité, la mémoire et la prière, et nous éloigne ainsi de la littéralité ${ }^{15}$ comme grille de lecture, pourvu que nous soyons conscients des enjeux et innovations celaniens qui alimentent la poésie de Tellermann. ${ }^{16}$

Au début de cette première section, Tellermann met en évidence le contact qu'elle veut garder avec Celan, la compréhension qu'elle veut avoir de sa parole et le dialogue à instaurer avec les persécutés et les abandonnés. Les allusions qui vont dans ce sens-là foisonnent par la suite, en particulier la possibilité que ce soit lui qu'elle « cache dans la pulsation » (30) et qu'ils souhaitent tous les deux réconcilier l'humain et le cosmique à travers des « univers chuchotés » (63). Les premiers mots - « Fatigue [...] claie [...] formules » (17) - évoquent

Rainer Maria Rilke, Les Élégies de Duino. Les Sonnets à Orphée, trad. Armel Guerne, Paris : Seuil, 1974, Sonnets 1.5, p. 111 : « Laissez la rose / chaque année [...] seulement fleurir. » Voir aussi Rainer Maria Rilke, The Selected Poetry of Rainer Maria Rilke, ed. and trans. Stephen Mitchell, New York :Vintage, 1989, « Uncollected Poems 1913-1918», p. 142-43 « Ausgesetzt auf den Bergen des Herzens » / « [Exposed on the cliffs of the heart] ». Cf. Paul Celan, « Corona », [Pavot et Mémoire], Choix de poèmes, op. cit., p. 49 et p. 51 : « Il est temps que la pierre se résolve enfin à fleurir », qu'à l'inquiétude qui nous anime «batte un cœur ». Jean-Marie Gleize, « Esther Tellermann », op. cit., p. 816.

16 Cf. Paul Celan, Enclos du temps / Zeitgehöft, op. cit., p. 9, p. 13 et p. 25, à propos des chants «brisé [s] », de «la prière / contre la prière » et du temps « qui résiste à la folie », ainsi que, à l'égard des « formules », les poèmes «J'ai entendu dire... », [De seuil en seuil], et « Give the Word », [Renverse du souffle], Choix de poèmes, op. cit., p. 83 et p. 275-77 (275). 
les troubles psychiques et les «formules » énigmatiques prononcées comme mots de passe dans le poème « Give the Word $»,{ }^{17}$ ainsi que le topos celanien d'une grille de parole avec laquelle s'orienter dans la neige du souvenir, voir autrement nos semblables et mieux explorer le dire :

\author{
Fatigue. \\ Je veux dire \\ claie pour s'enfoncer \\ dans la lecture des formules. \\ Malgré le système des miroirs \\ il me fit défaut : \\ « Le Prince avait absorbé les images. »
}

Un ton familier et peut-être même de l'espièglerie se dégagent des expressions « Je veux dire » et " Malgré le système des miroirs », et pourtant il est clair que bâtir un Nous sera primordial. Il est intéressant de comparer ces vers à quelques textes d'Enclos du temps, où des « maximes » se tortillent, des « lignes d'images » sont gardées à l'abri et le « deuil » passe dans le camp d'un autre. ${ }^{18}$ Le chagrin, le déséquilibre et le désarroi font partie intégrante des textes poétologiques de Celan, où " [1]es images » renvoient souvent aux bien-aimés perdus, aux innocents « fauch[és] » par un dieu impénitent. ${ }^{19}$ Dans Guerre extrême, les énoncés " Malgré le système des miroirs » et « Le Prince avait absorbé les images » ont beau être énigmatiques, ils suggèrent de par leurs allusions le deuil prolongé de Celan, sa parole de bénédiction ${ }^{20}$ et les écueils auxquels il faut faire face. Il s'agit d'un dialogue avec cet Autre, en tant qu'être couronné de souffrance mais qui rend ainsi la parole plus vraie, ${ }^{21}$ et de poursuivre ce dialogue même si l'on se heurte à des difficultés passagères.

Il est également important d'aborder les rites patients et souvent allusifs qui, malgré les titres des deux séquences, «Terre mentale » (13-86) et « Guerre

17 Paul Celan, « Give the Word », [Renverse du souffle], Choix de poèmes, op. cit., p. 275-77 (275).

18 Paul Celan, «Dans la plus lointaine » et «Toutes les formes du sommeil », Enclos du temps /Zeitgehöft, op. cit., p. 25 et p. 29.

19 Cf. Paul Celan, «Une étoile », Enclos du temps / Zeitgehöft, op. cit., p. 53 : « son dieu / fauchant passe en revue le front des images ».

20 Ibid., «Une étoile » et « Baigné de lumière les germes », p. 53 et p. 97 : « ta sanglante / salive / bénit / un grain de poussière possédé »; « une parole de bénédiction, en avant, / se serre, / poing / sensible au temps ».

21 Cf. Paul Celan, «Psaume», [La Rose de Personne], Choix de poèmes, op. cit., p. 181 : « la couronne rouge / du mot pourpre que nous chantions, / au-dessus, ô, au-dessus / de l'épine». 
extrême » (87-147), adoucissent la quête de sens. Reprenons, par exemple, le motif de l'errance, du poète comme des disparus. Le fascinant chez Tellermann, c'est l'amour avec lequel elle aborde ce topos, l'humilité et le ton humble adoptés dans son rapport aux guerres de l'histoire. Lire ces poèmes dans une optique dialogique, c'est observer un voyage dans le temps et l'espace, y participer, arpenter avec l'Autre un monde mi-rêvé, mi-réel (cf. 18, 59, 61, 86). De nombreuses allusions font de Celan cet Autre, en tant que celui avec qui refaire la Parole, rebâtir des lieux au moyen de mots ciselés et de silences, traverser des pays du cœur désormais désertés et les repeupler, y dresser « les tentes » $(59),{ }^{22}$ y sentir « l'odeur » $(9,59,86)$ qui vient du dehors comme du dedans et qui nous initie $(133,136)$, se baigner dans « [l] es fleuves » $(86 ;$ cf. 19, 64) qui nous situeront dans un renouveau constant héraclitéen. ${ }^{23}$ Le leitmotiv « un trou d'étoiles » $(102,130,132,141)$ qui lui est emprunté ${ }^{24}$ souligne le désir de communiquer avec ceux et celles à qui la langue et la vie ont été volées, ainsi que le souffle renouvelé que l'on respire auprès d'eux au sein d'une parole retrouvée. Tellermann « roul[e] » comme Celan « les autels vers l'intérieur du temps $»^{25}$ : elle prépare « la table des sacrifices » (47). Or, elle s'occupe de son aîné en même temps, prend soin de lui et fait donc avancer la parole commémorative. Elle lui tend la main dans « Terre mentale » en se référant à divers paradigmes : les « dates » de l'événement, à ne jamais oublier $(33,43)$; la blancheur du néant qui sépare les exilés de nous comme un «voile», tout en les réunissant et leur permettant de gravir les temps et les âges $(35,74,75,77)$; la parole de l'après-guerre comme écriture syllabique où doit battre le cœur des absents $(41 ; \mathrm{cf} .122)$; et l'alliance qui ne cesse de se creuser au travers de prières quotidiennement reprises $(19,42) .{ }^{26}$ Il s'agit de traverser le cosmos, avec autant

22 Ibid., «En l'air là-haut... », [La Rose de Personne], p. 223 : « les divisés avec leurs / bouches éblouies », « les dispersés », « les étrangers à vie », « les / errants conduits à travers le désert stellaire Âme », qui sont tous des « dresseurs de tentes là-haut dans l'espace ». Voir aussi p. 199-201 (201), « Anabase », quant au « mot-tente qui devient libre : / / ensemble ». Ibid., «Dans les fleuves », [Renverse du souffle], p. 229, les vers « Dans les fleuves au nord du futur, / je lance le filet ». Cf. « Argumentum e silentio » [Pour René Char], De seuil en seuil, p. 111-13 (111), où il s'agit de déposer des mots qui chantent pour les disparus, notamment le mot créé implicitement par la mer du temps, 'des' temps et des pleurs ; et « Tas de coquillages ", Contrainte de lumière, trad. Bertrand Badiou et Jean-Claude Rambach, Paris : Belin, 1989, p. 15, où l'on est emporté par des fleuves vers un temps annonciateur du renouveau de la parole et de retrouvailles avec les désertés.

24 Paul Celan, « Projeté », Enclos du temps / Zeitgehöft, op. cit., p. 27.

25 Ibid., « Des lunes haineuses », p. 11.

26 Quant aux dates, voir Paul Celan, Le Méridien \& autres proses, trad. et éd. Jean Launay, Paris : Seuil, 2002, p. 71, p. 73, p. 106n46 et p. 107n5o, ainsi que la discussion des dates comme évocation de l'événement dans Andréa Lauterwein, Paul Celan, Paris : Belin, 2005, 
d'ambition et de chaleur que de retenue et de respect. Au niveau socioculturel, ces textes et les rites auxquels ils font référence dépassent ainsi - avec tendresse - la reprise de formes poétiques à la mode en France pendant ces dernières décennies.

L'eau y joue un rôle protéiforme, comme pour dire que l'énigme nous entoure et que nous n'avons qu'à accueillir sa présence dans nos représentations. Elle évoque la mort et la vie, la création et la disparition, le temps qui passe et l'unité des espaces distincts, notre vécu intersubjectif et des traces à suivre dans l'inconscient pour mieux infléchir les rapports à venir. Ce motif apparaît à maintes reprises et, à l'instar de Protée dans la mythologie, suggère la possibilité de saisir l'avenir mais sans forcément répondre à nos questions. Par exemple, le Je lyrique tient les autres et le dehors " comme un fleuve », notion paradoxale qui nous réconforte de par son étreinte des horizons, tout en impliquant paradoxalement et par les répétitions - « vallées / murs / où fixer / où fixer les journées » (19) - que nulle journée ne doit trouver le repos, sauf peut-être dans la pensée. L'eau fait écho au besoin de réconcilier des contraires selon le rite. Le Je lyrique « transcri[t] » ces « sables » mouvants, au fur et à mesure que leurs ondulations adviennent et sont perçues. Le dialogisme entre en jeu dans la mesure où l'on habite l'« eau mentale / feu » (70) d'autrui, suivant les méandres de l'inconscient et de l'intersubjectif, creusant les «nappes souterraines » (145) où l'alliance peut advenir et nous illuminer. Les répétitions tout au long du recueil inscrivent la durée dans ce propos, signalant les différentes « vitesse[s] » (86) du songe, sa façon imprévisible mais insistante de nous instruire quant à l'événement. Chaque « rive bue » $(21 ; \mathrm{cf} .71,74,147)$ nous met « au bord de » quelque chose qui n'est jamais vraiment précisé ( 36 ; cf. 144, 147), sinon la richesse de la « soie » que seront les fils narratifs et langagiers (74; cf. 112, 133), « l'ampleur de la mer » (147) qui atteindra nos cinq sens. De même, les points de repère mentionnés - « Nil et Neva » (22) en Afrique et en Russie, « le Gange » (76) en Inde et au Bangladesh - ne nous ancrent que dans l'antique et l'universel, le «sacré » immémorial « où vivent bêtes et gens » (69), les grands espaces que délimite le chiffre 4 (51, 89, 90, 114, 129, 131, 142, 144). Ces traversées ont lieu « en fièvres / de cénotaphe en cénotaphe » (64).

p. 8-10. L'image d'une « écriture syllabique » vient du poème « Moi aussi », Contrainte de lumière, op. cit., p. 151 ; voir aussi « Argumentum e silentio » [Pour René Char], De seuil en seuil, op. cit., p. 111-13, à propos de syllabes transpercées par une dent à venin et du devoir de gravir le temps pour témoigner même si le témoignage paraît presque impossible. Quant à l'image d'un « voile », puis de prières quotidiennes où les juifs très pratiquants sanglent deux petites boîtes en cuir au bras gauche et sur la tête, voir Choix de poèmes, op. cit., p. 87 («Lointains », [De seuil en seuil]) et p. 245-47 (« Dans la lanière de prière blanche », [Renverse du souffle]) ; cf. Choix de poèmes p. 365nı et GE 42, « vos sangles ». 
Il s'agit en somme d'errer aux côtés de l'Autre et de se souvenir avec lui plutôt que de résoudre des dilemmes (cf. 142), que cet Autre soit celui qui témoigne de la guerre ou tous ceux, plus indéfinissables, qui « cherch[ent] refuge » et ne sont «qu'un » (126). L'eau nous aide à comprendre l'énigme comme partie intégrante de la démarche commémorative tellermannienne.

Il convient ensuite d'analyser deux aspects techniques qui enrichissent Guerre extrême tout au long des deux séquences: d'autres images qui lui donnent un aspect cérémonial, puis la façon dont le maniement du lexique fait penser aux Psaumes. Considérons des moments épars, regroupés par thème. L'ambiguïté spatiotemporelle, par exemple, va de pair avec l'envie de relier des versants du réel et de l'Histoire. Dans ce « faste des choses tues » $(115,132)$, les normes logiques d'un exposé raisonné ne peuvent s'appliquer. Les profondeurs du rêve prennent le dessus, dans le but d'ouvrir notre regard sur ce qui nous soude malgré l'événement. Cela permet à l'auteur de privilégier des « superpositions » $(28,117)$ inattendues, ce qui en augmente peut-être l'opacité, mais suggère néanmoins la reprise cyclique de gestes visant la consolation personnelle et collective, le tissage d'une étoffe riche en symboles et la semaison de graines langagières qui alimenteront la pensée et l'affect. Ainsi l'idée d'être «derrière le monde » (114), image empruntée à Celan ${ }^{27}$ qui reprend d'autres fils conducteurs et s'insère bien parmi les autres fragments du récit. Tellermann nous guide vers ce point de mire, afin que l'on voie comme voient les morts et rêve comme eux de faire pleinement partie d'un tout, en osmose avec le cosmos, l'élémentaire, le renouveau. De façon performative, elle réintroduit la dénudation «jusqu'au temps » et les limites des « [e]spaces trop courts » annoncées au début $(10,12)$. Bien que « clos » $(94,114)$, les symboles s'ouvrent paradoxalement, comme pour commencer à fleurir, au fur et à mesure que tel ou tel motif ressurgit ou s'adapte aux contours de la narration. De plus, ils sont souvent liés aux êtres humains et aux rencontres, telle l'énigmatique « Femme » à la même page - la locutrice ? une bien-aimée ? - qui « préciserait / la rosée / vous dresserait / vous et plusieurs » (114). Toutes sortes d'énoncés rythment ainsi le récit. Pas vraiment explicités, ils jouent néanmoins un rôle important, contribuant aux cérémonies proprement dites et rendant encore plus somptueux le faste

27 Paul Celan, «Je te pilote», Enclos du temps / Zeitgehöft, op. cit., p. 49 : «Je te pilote derrière le monde, / te voilà chez toi, inébranlable »; «Un anneau, pour tendre l'arc », p. 67 : «Un anneau $[. .$.$] envoyé derrière un essaim de mots / qui se précipite derrière le monde ».$ Cf. Enclos du temps, p. 11 (« Des lunes haineuses ») et p. 25 («Dans la plus lointaine »), à propos d'être « derrière le néant » et «derrière la pointeuse », puis Choix de poèmes, p. 223 («En l'air là-haut», [La Rose de Personne]) et p. 235 ("Soleils-filaments », [Renverse du souffle]), quant à l'idée d'être « là-haut dans l'espace » parmi « les étrangers à vie », « au-delà des hommes». 
entraperçu de la communion avec les disparus, d'hymnes qui leur sont chantés, du déploiement du sens.

Certaines images nous font célébrer le monde et autrui à travers des rites immémoriaux. Dans ce livre qui « serait / un et plusieurs » (108), l'un des fils incontournables est celui des soins apportés aux êtres inapaisés, que ce soit des morts d'autrefois ou des communautés de vivants de toute ère conscients de leurs semblables et du poids de l'existence. Il en résulte dans Guerre extrême des motifs qui nous font ressentir de la chaleur humaine malgré tout, dans l'attente de meilleurs jours comme le ferait les Psaumes. Sont retenus « en alternance » $(123$; cf. $73,117,138)$ ombre et lumière, ce qu'il advient de lourd (cf. $116,122,125,132)$ et ce qu'il y a de léger que l'on peut « recueillir » et « porter » $(119,120)$. Ces « épopées » modernisées sont certes assez sombres lorsqu'il est question de « collines » devenues « fosses » (111, 115), mais nous font goûter l'ouvert et la déférence envers le repos « sous le cri des oiseaux » et les « lèvres à jamais / en épiphanies » (50). Se présente assez tôt le besoin d'ensevelir (26), mais sont mentionnés aussi des gestes plus quotidiens : la génuflexion, la prosternation, les soupirs $(35,115,124)$. Le sens rayonne lorsque les motifs fragmentaires s'intriquent, par exemple là où la génuflexion se veut peut-être manière de «porter le soleil » ou de se faire accordé par la suite les «sucs » des « troupeaux » et des « voix étrangères » (35), puis lorsque l'énoncé « les ors parallèles » (106; cf. 108, 110, 125, 138) insiste sur l'émerveillement que nous inspirent les gestes et histoires qui nous inscrivent dans la durée. S'adressant très probablement à Celan, Tellermann montre que l'émerveillement peut jaillir de sons « frappés » et de leur « silence » (91), de méditations tâchant de se trouver une place parmi les « litanies » difficiles à assimiler (107) ou des ors «qu'allument les rouleaux» (106), peut-être ceux de la Torah dans la tradition juive. À travers ces reprises et juxtapositions, le livre devient en somme l'un des « temples » $(18,117)$ où l'on continue à espérer - comme le dirait Tellermann, où la quête de sens accumule des offrandes dans un feu sacré qui « brûl[e]/ toute déambulation / en symbole » $(71 ; \mathrm{cf} .77,83,124)$, qui transforme les « promenades » (86) avec l'Autre en découverte du potentiel de transformation de soi et de l'ordre symbolique que communiquent des motifs polyvalents.

Au plan technique, les structures qu'emploie Tellermann - les répétitions, les ellipses, les épaisseurs lexicales - font réellement des poèmes de Guerre extrême des « psaumes détournés / dans l'attente » (107). Lues par le biais de la tradition biblique, en tant quécho d'aspects des Psaumes, elles sont au cœur du caractère insituable du récit tellermannien. Par ailleurs, les Psaumes incarnent un élan quasi sans âge vers le cosmos. Leur élan poétique a des racines dans le Proche-Orient datant de 1600-1200 avant l'ère commune, précédant la rédaction des psaumes de l'Ancien Testament aux alentours de 1000-400 avant l'ère 
commune. Comme nous le rappelle la critique, il s'agit de chants liturgiques à mettre en musique qui servent à louer ou à dialoguer avec un Dieu non seulement du cosmos, mais aussi de l'histoire. Le divin n'y est pas forcément le religieux. On peut faire la typologie des 15 o chants, mais le dialogue qui s'y engage est autant avec le sacré qu'avec le langage et avec soi, pour célébrer la création et «ne pas être seul au monde ». ${ }^{28}$ Il faudrait savourer les cadences et les symétries du dire, liées à une voix « à la fois anonyme et universelle »- d'auteurs différents - qui traverse si bien les siècles et les traditions littéraires, ${ }^{29}$ autant que les profondeurs, les abîmes et les défis dont parle le poème.

Il convient à cet égard de citer très brièvement Psaumes : Traductions 19181953 de Paul Claudel (1868-1955), afin de montrer quelques thèmes et formes caractéristiques. Cela nous permettra de voir l'actualité de la Bible - dans ses dimensions affective, sociale, existentielle, langagière - vis-à-vis du poème tellermannien. À condition de ne pas confondre la foi enthousiaste de l'un et le pas solide mais momentanément alourdi de l'autre, cette mise en contexte peut porter ses fruits. Dans les deux cas, l'être humain lutte avec soi et avec l'histoire, est en dialogue avec la création, se met aux aguets au cas où le changement s'annoncerait à l'échelle intime ou collective. Pour Guy Goffette, les psaumes sont

28 Henri Meschonnic, Gloires : traduction des psaumes, Paris : Desclée de Brouwer, 2001, p. 22, ainsi que p. 20 à l'égard d'« une subtile dissociation entre le divin et le religieux ». Nous nous appuyons aussi sur les ouvrages suivants : Collectif, La Bible, Segond 21, Genève : Société Biblique de Genève, 2007, p. 369 ; Nahum M. Sarna, On the Book of Psalms : Exploring the Prayers of Ancient Israel, New York: Schocken, 1995, p. 3-23, p. 205-07; Robert Alter, The Book of Psalms, op. cit., p. xiii-xviii ; Robert Alter, The Art of Biblical Poetry, Revised Edition, New York : Basic, 2011, p. 151-70, notamment p. 151, à propos d'un « God of history » (Dieu de l'histoire), et p. 170, concernant la prise de conscience dans les Psaumes « of the linguistic medium of religious experience » (des moyens linguistiques par lesquels se communique l'expérience religieuse) ; Robert Alter, The Art of Biblical Narrative, New York : Basic, 2011, p. 226-28, vis-à-vis de la manière dont les récits bibliques gravitent généralement vers le dialogue, et ainsi, au passage, vers des commentaires obliques; et Hermann Gunkel, The Psalms : A Form-Critical Introduction, Philadelphia : Fortress, 1967, en particulier à propos de classements.

29 Marie-Josette Le Han, « La structure liturgique dans Une somme de poésie de Patrice de La Tour du Pin », Poésie et Liturgie : XIX ${ }^{e}-X X^{e}$ siècles, éd. Martine Bercot et Catherine Mayaux, Oxford : Peter Lang, 2006, p. 153-65 (159). Cf. Robert Alter, The Art of Biblical Poetry, op. cit., p. 139-42, et The Book of Psalms, op. cit., p. xiii. Chantal Labre, Dictionnaire biblique culturel et littéraire, Paris : Armand Colin, 2002, p. 246-47, discute par exemple des traductions de Corneille, La Fontaine, Marot, Racine ; de la paraphrase des psaumes au XVII siècle; de la célébration ou de l'écriture de psaumes chez Huysmans, Claudel et Patrice de la Tour du Pin; et de l'emploi par Hugo du mot «psaume » pour indiquer ce qui est sombre, religieux, ténébreux. 
faits « pour vivre aujourd'hui, louer, crier, pleurer, prier ».30 Selon Claudel, il n'y a pas besoin de comprendre pour vouloir écouter, répondre à David, Asaph et d'autres, « y mettre du sien », respirer comme le font les versets bibliques et leurs « personnages mystérieux ». ${ }^{31}$ Au niveau lexical, notons chez lui vis-à-vis de la Bible et comme dans Guerre extrême la diversité de tons, le recours fréquent au vocabulaire élémentaire du corps et du dehors et la fascination pour le feu et l'éternité comme reflets des mystères du temps - pour Claudel, ceux également du Créateur. Certains passages chez Claudel éclairent d'ailleurs Guerre extrême, soulignant comment Tellermann opte pour le pianissimo et l'étonnement, détourne la louange en racontant l'attente pleine d'espoir mais tout de même prolongée, diminuée par la gravité de l'événement. En contraste avec l'« océan de bouches ouvertes qui crient » pour applaudir Dieu chez Claudel, ${ }^{32}$ la locutrice de Guerre extrême, en train d'accompagner Celan, nous semble-t-il, remarque plutôt la tristesse des exilés auxquels la vérité de parole fut volée : « Bouches me rapportent / aux eaux saumâtres / et voguions / "vers un trou d'étoiles" » (141). Chez Claudel, des répétitions insistantes soulignent la puissance du Créateur, qui prend parfois la figure du Soleil surveillant toute Sa création. Psaume 67 se moque des ennemis de Dieu, de leur faiblesse et de leur risible impuissance: «Comme de la poussière, comme la poussière en tourbillons qui s'envole ! / Comme la fumée qui se dissipe, dissipe-les, Seigneur, / Comme la fumée qui fond au feu, fais-les fondre, Seigneur $! »^{33}$ Cet exemple pris au hasard en dit long sur l'elliptique, l'élémentaire et l'exploratoire chez Tellermann, du moment qu'il est lu en miroir avec le texte suivant de la deuxième séquence :

Feu

fut porté.

Sable

fut porté.

Nous avions parcouru

nuit.

Tubéreuses d'où nous vînmes. (119)

$30 \quad$ Paul Claudel, Psaumes : Traductions 1918-1953, éd. Renée Nantet et Jacques Petit, avant-propos de Pierre Claudel, préface de Guy Goffette, Paris : Gallimard, 2008, $4^{\mathrm{e}}$ de couv.

$31 \quad$ Ibid., p. 20. Goffette parle à son tour dans la préface, « Une feria claudelienne », p. 7-10 (7), de l'envie que l'on peut avoir de s'y mettre : « Pas besoin d'être écrivain, ce n'est pas de littérature qu'il s'agit, mais de respiration. »

$32 \quad$ Ibid., Psaume 46 [Psaume de l'Ascension], p. 141-42 (141).

33 Ibid., p. 166-69 (166). Cf. Collectif, La Bible, Segond 21, op. cit., Ps. 67, deuxième verset, p. 389, un psaume de huit versets, en contraste avec les quelques pages chez Claudel: «Que Dieu nous fasse grâce et nous bénisse, qu'il fasse briller son visage sur nous ! 
Dépouiller ainsi le dire, c'est célébrer l'essentiel dans la parole et dans les rapports humains, mais en même temps prendre du recul et se demander si nous sommes réellement sans appui. Le constat prend le dessus, en langue aussi allitérative et oraculaire que chez Claudel mais réduite au minimum, se faisant l'écho non pas uniquement de la parole de Dieu mais aussi de celle d'âmes errantes, acheminantes, réunies mais jamais arrivées. Cette comparaison confond certes les genres en confrontant la louange à la lamentation, mais elle donne un aperçu des Psaumes en tant que source poétique dans laquelle puiser.

Alors que Claudel lisait les Psaumes en latin, ${ }^{34}$ Celan roulait « les autels / vers l'intérieur du temps $»^{35}$ par le biais de l'allemand, de la culture judaïque et d'un face-à-face avec l'exil. S'appuyant sur sa connaissance intime de l'hébreu et des horreurs de l'Histoire, il se souvient des psaumes dans certains de ses textes particulièrement connus, mais détache ceux-là de leur fonction originelle. ${ }^{36}$ Tandis qu'un Claudel se réjouit sur les plans sonore, lexical et sémantique de la gloire du Seigneur, Celan ne conforte nullement la foi. Notamment dans « Fugue de mort », qui évoque le psaume 137, puis aussi dans le poème «Il y avait de la terre en eux », il privilégie les qualités consonantiques de l'allemand - et pense très probablement à la compacité de l'hébreu - pour tourner en dérision la cruauté et l'absurde de la Shoah. Que faire pour habiter un monde aussi brutalement désacralisé que celui dépeint dans ces textes? Voilà une question à laquelle Guerre extrême tâche d'apporter des réponses en réconciliant le passé et le futur, la souffrance de Celan et l'en-avant de la parole poétique, la tradition lyrique et un chant réinventé à rebours échappant

\footnotetext{
34 Ibid., p. 128n1.

35 Paul Celan, « Des lunes haineuses », Enclos du temps / Zeitgehöft, op. cit., p. 11. Cf. GE 97 : «Guerre est filet / vers l'intérieur du temps. »

36 Voir Andréa Lauterwein, Paul Celan, op. cit., p. 57-61, son analyse des poèmes « Fugue de mort » de Pavot et Mémoire (op. cit., p. 83-89, poème que nomme Lauterwein « Fugue de la mort ») et «Il y avait de la terre en eux » de La Rose de Personne (trad. et postface Martine Broda, Paris : Seuil, 2007, p. 11). Selon Lauterwein, « Fugue de mort » inverse la fonction anamnétique du psaume 137, et « Il y avait de la terre en eux » nous renvoie aux psaumes 115, 107 et 98 mais les détourne en faisant sombrer le chant, la langue et l'alliance avec Dieu. À propos de l'action de creuser comme le font ceux dans ces deux poèmes et de s'avancer vers Dieu à rebours (en creusant leurs propres tombes comme dans « Fugue de mort », creusant vers «nulle part » comme dans « Il y avait de la terre en eux »), voir aussi GE 97 : « Guerre est filet / vers l'intérieur du temps. / / Ils bâtissent le Nom / avec pelles râteaux. » Tellermann cite « Il y avait de la terre en eux » en entier dans une brève notice consacrée à Celan et à « une langue autre » : Esther Tellermann, « Poésie », op. cit., p. 5 o. Enfin, voir aussi la référence aux psaumes d'autrefois dans « Strette », Grille de parole, trad. Martine Broda, Paris : Seuil, 2008, p. 103.
} 
aux « boues » $(96 ; \mathrm{cf} .64,142) .{ }^{37}$ Le poème suivant, tremplin pour la présente analyse, met en évidence les psaumes en tant qu'arrière-plan de la poésie de Tellermann d'une part, et, de l'autre, renvoie au fait que Celan avant elle les a admirés puis détournés :

De la sorte vous laisseriez la trace
des réminiscences
couronnes comme pneus
corps entravés en langueur
$\quad$ Vous
$\quad$ diriez
l'enclave dans leurs litanies
psaumes détournés
dans l'attente. (107)

Pensons encore une fois au caractère aberrant et fou de l'événement dont le poème creuse les traces, les sillons de sang et de cendres, en contraste avec le caractère litanique de maints textes sacrés. Les corps de la guerre entravent le dire mais en sont aussi l'enclave, le lieu d'où peut émaner le souffle poétique. Pensons à ce que Celan appelle « le dedans de la roche »,38 enclave d'où peut surgir la confiance de celui qui attend et espère. N'oublions pas l'air dans ces couronnes absurdes " comme pneus », couronnes plutôt de foire ou pour ne pas se noyer ayant des liens de parenté avec ce que Martine Broda nomme «la Couronne ironique et tragique du Dieu absent » $(\mathrm{cf} .85,99) \cdot{ }^{39}$

Ce texte contient aussi quelques structures qui nous permettront de considérer la manière dont Tellermann emprunte aux psaumes une étoffe poétique riche en reprises et parallélismes, et ainsi plus dense, polysémique et atemporelle. Réduire Guerre extrême à un palimpseste des Psaumes n'est pas notre but. Bien au contraire, la comparaison met en lumière un goût pour la concision, les motifs, la musicalité d'un chant brisé et les couches de sens qui

37 Cf. GE 142, référence possible à Celan : « Eaux fendues / tournent autour / de celui / qui scella / une langue / en 4 boues », c'est-à-dire celui qui établit une voie, creusa un sillon, créa un possible, imagina les paysages où s'annonce une renverse du souffle, le pays de la contre-parole que d'aucuns appellent « la Celanie », néologisme de la part de l'auteur (voir Andréa Lauterwein, Paul Celan, op. cit., p. 21). Voir aussi GE 41 : « Tu es couché / dans le battement d'une syllabe».

38 Paul Celan, «Confiance », [Grille de parole], Choix de poèmes, op. cit., p. 131.

39 Paul Celan, La Rose de Personne, op. cit., p. 174. Cf. le paradigme du souffle, de la poésie dite «pneumatique », de l'air qui circule et par lequel on se lie à l'Autre et réapprend à habiter le monde. 
s'approfondissent au fil des pages. Qui plus est, ce détour par la stylistique révèle des aspects par lesquels son chant se distinguera par la suite, dans des recueils de plus en plus développés et dont les épaisseurs intéresseront la critique. Les répétitions, d'abord, comme celles que nous avons vues précédemment à la fois sonores et sémantiques : les consonnes liquides [l] et [R] qui parcourent le texte, y ajoutant des traces, des langueurs; la consonne [k] des mots couronnes, corps et enclave, mots par lesquels on descend de haut en bas, du dehors vers le dedans, de l'apparence vers l'affect, la matière, le souffle; le son [t] par lequel les litanies se transforment en attente. Les reprises, ensuite, où un élément clé qui porte sens se trouve légèrement modifié au niveau morphologique, sémantique ou phonétique, tels les mots entravés et enclave, litanies et psaumes, trace et attente.

Dans ces exemples, c'est la cohérence des séries qui frappe. Fond et forme sont si intimement liés, menés à bien de façon si lucide, qu'il ne suffirait pas de réduire le tout au lyrisme, à la muse. Les nombreuses spécificités de l'hébreu dépassent le cadre de notre comparaison, mais il est possible de jeter un œil sur Gloires d'Henri Meschonnic pour en avoir une idée, à savoir du «primat de la rythmique » et de la brièveté des unités de sens, évidents dans sa version du premier verset du psaume 137: «Sur les fleuves de Babylone où dans nos chaînes oui dans nos larmes / / Nous nous souvenions de Sion ».40 Notons en particulier la quasi-répétition du syntagme « où dans nos chaînes », ainsi que la reprise sémantique «nos chaînes [...] nos larmes » et la reprise du son [s]. Il s'agit de composantes faciles à moduler qui servent aussi d'aide-mémoire. Tellermann, pour sa part, emploie régulièrement des composantes reprises et légèrement modifiées, en série ou sous forme de motifs, dont le sens ne cesse d'éclater et de se refaire, de sombrer et de s'illuminer, de s'adapter aux contours d'un récit concernant une attente toujours prolongée, que ce soit en blocs complémentaires comme « coût / et prière » (62) ou en cascades plus énigmatiques et polysémiques qui s'ouvrent sur le temps et l'espace : « fleur

$40 \quad$ Henri Meschonnic, Gloires, op. cit., p. 37, p. 342. Psaume 137 sous la plume de Robert Alter est particulièrement concis, autrement rythmé : «In Babylon's streams, / there we sat, oh we wept, / when we recalled Zion » (Dans les fleuves de Babylone, / là nous nous assîmes, ô nous pleurâmes, / en nous souvenant de Sion) (The Book of Psalms, op. cit., p. 473). Dans The Art of Biblical Poetry, op. cit., p. 116-22, Alter s'intéresse à la concision, vis-à-vis de l'hébreu, de la traduction de la Bible faite par Martin Buber et Franz Rosenzweig, traduction qui respecte dans la mesure du possible la répétition dynamique de mots ou de mots-racines. La répétition de chaque Leitwort (mot ou mot-racine récurrent) surgit du tissu sonore et sémantique et amplifie le rythme intérieur du texte. Celan lisait cette traduction, et il se peut donc que Tellermann absorbe en partie à travers lui cette approche de la langue poétique. 
/ écriture / stèle / enlacement » (9o). À l'instar des Psaumes, elle reprend des énoncés, des mots et des unités de souffle pour accentuer un processus d'acheminement, celui à la fois de la pensée, de l'inconscient au moyen du rêve et d'une locutrice en pleine promenade avec l'Autre (cf. 86). Comme dans la version hébraïque des Psaumes, les nuances ajoutées au récit par les parallélismes au sein de courts versets évocateurs rendent le sens plus fluide, nous invitant à continuer à les méditer. ${ }^{41}$

En guise de conclusion, rappelons-nous que les Psaumes contiennent des hymnes concis et hautement métaphoriques, qui nous soutiennent de par leurs chants tantôt ténébreux, tantôt ensoleillés, voire les deux en même temps. Guerre extrême nous amène sur des chemins semblables. On se met à l'écoute de prières énigmatiques vers autrui. On se ressouvient de la portée de la langue poétique, ainsi que de ses écueils. On apprend à lire autrement, selon une rythmique singulière censée nous montrer l'urgence du dire et le rôle qu'y joue le témoignage. Même si Tellermann se met en dialogue avec Celan pour soigner la parole et contempler l'Histoire, sa prise en compte de la finitude humaine et son utilisation des formes littéraires s'inscrivent dans une continuité, alimentent la tradition autant que la modernité. Son chant a beau se briser, se dépouiller, il n'en ressemble pas moins dans sa gravité à celui d'autres voix majeures, par exemple celui de Claude Esteban (1935-2006) « tourné vers le sensible qui peut apaiser le corps accueillant la blessure de l'Occident » $(N N$ 42 ; cf. GE 51), ou bien de Philippe Jaccottet (1925-) à la recherche d'« une vie à l'intérieur de la vie $»^{42}$ Son récitatif rejoint également les voies de l'allégorie médiévale : «Lire, c'était chercher la vérité qui nous échappe, c'est-à-dire pratiquer l'allégorie », en particulier celles, intransigeantes, qui traitent d'une vérité complexe et dont la nature insaisissable donne la mesure de sa valeur. ${ }^{43} \mathrm{Il}$ s'agit d'un sacré là devant nos yeux mais dont on peut avoir du mal à parler, tant le

$41 \quad$ Voir aussi Robert Alter, The Book of Psalms, op. cit., p. xx-xxiv, et S. E. Gillingham, The Poems and Psalms of the Hebrew Bible, Oxford: Oxford UP, 1994, chapitre 4, p. 69-88, « Parallelism» (Parallélisme).

42 Vis-à-vis de problématiques similaires à celles qu'aborde Tellermann dans Guerre extrême, voir par exemple Philippe Jaccottet, "Sur les pas de la lune », L'Encre serait de l'ombre, Paris : Poésie/Gallimard, 2011, p. 63-66 (66) : « un espace émané de ce monde et pourtant plus intime, une vie à l'interieur de la vie » ; « Oiseaux, fleurs et fruits », ibid., p. 135-56 (143): « Je crois que j'ai bu l'autre monde ».

43 Frank Talmage, «Apples of Gold :The Inner Meaning of Sacred Texts in Medieval Judaism », Jewish Spirituality: From the Bible through the Middle Ages, ed. Arthur Green, New York: Crossroad, 1987, p. 313-55 (318) (nous traduisons). À propos d'allégories profondes et intransigeantes, où les rapports et un processus comptent plus que l'exposition (« [where it is a matter of] relationships and process rather than statement »), Talmage cite Gay Clifford, Transformations of Allegory, London/Boston : Routledge and Kegan Paul, 1974, p. 53. 
langage courant semble y résister, le deuil nous affaiblir. Constatons enfin le courage qui sous-tend Guerre extrême, où chacun est « anneau » pour l'Autre, «pour toujours » intéressé par l'alliance, par la complémentarité des êtres et des voix qui nous vient de divers versants dès que nous « avan[çons] » (GE 145). C'est ainsi que Tellermann nous hèle des « rives » (147) qui nous habitent: en affirmant que cela vaut la peine de tendre la main à l'Autre, afin de se souvenir, d'avancer, de réconcilier. 